\title{
COMPARAÇÃO DOS NÍVEIS DE APTIDÃO FÍSICA ENTRE ESCOLARES PRATICANTES E NÃO PRATICANTES DE JIU-JITSU
}

\author{
COMPARACIÓN DE LOS NIVELES DE APTITUD FÍSICA ENTRE LOS ESCOLARES \\ PRACTICANTES Y NO PERTENECIENTES A JIU-JITSU
}

\author{
COMPARISON OF PHYSICAL FITNESS LEVELS AMONG PRACTICING AND NON- \\ JIU-JITSU SCHOOLCHILDREN
}

\author{
Carlos ALBERTO DA SILVA ${ }^{1}$ \\ Abraham Lincoln de PAULA RODRIGUES ${ }^{2}$ \\ Stela LOPES SOARES ${ }^{3}$
}

RESUMO: O jiu-jitsu é um esporte que vem crescendo bastante nos últimos anos. Se teve como objetivo comparar os níveis de aptidão física entre escolares praticantes e não praticantes de jiu-jitsu de um bairro na cidade de Fortaleza/CE. Foram analisadas as seguintes variáveis: IMC, Flexibilidade, Resistencia abdominal e Resistência cardiorrespiratória, com 22 crianças, todas do sexo masculino, divididas em 2 grupos com 11 indivíduos. Foi observado que o grupo jiu-jitsu se saiu melhor em todos os testes principalmente nos testes de flexibilidade (JJ:31( $\pm 2,00)$, EDF:28( $\pm 4,07))$ e resistência abdominal (JJ:30( $\pm 4,42)$, EDF:25( $\pm 2,99))$, nos quais obteve uma diferença significativa, já nas variáveis IMC(JJ:20( $\pm 2,02)$ EDF:22( $( \pm 4,28)$ e resistência cardiorrespiratória(JJ:654( $( \pm 127) \operatorname{EDF}(583( \pm 120))$ apesar de não existir diferença significativa, o grupo jiu-jitsu demonstrou valores superiores aos do grupo EDF. Com os resultados obtidos pode-se concluir que a prática do jiu-jitsu pode trazer benefícios a saúde tão bons ou melhores que a prática de educação física.

PALAVRAS-CHAVE: Aptidão física. Saúde. Educação física escolar. Jiu-jitsu.

RESUMEN: Jiu-Jitsu es un deporte que ha crecido mucho en los últimos años. El objetivo era comparar los niveles de aptitud física entre la práctica y los no practicantes de los estudiantes de Jiu-Jitsu en un vecindario en la ciudad de Fortaleza/CE. Se analizaron las siguientes variables: IMC, flexibilidad, resistencia abdominal y resistencia cardiorespiratoria, con 22 niños, todos varones, divididos en 2 grupos con 11 individuos. Se observó que el grupo de JiuJitsu era mejor en todas las pruebas principalmente en las pruebas de flexibilidad (JJ: 31 ( \pm 2,00), EDF: $28( \pm 4,07))$ y resistencia abdominal (JJ: $30( \pm 4,42), E D F: 25( \pm 2,99))$, donde obtuvo una diferencia significativa, ya en las variables IMC (JJ: $20( \pm 2,02)$ FED: $22( \pm 4,28)$ y resistencia cardiorespiratoria (JJ: 654 ( \pm 127$)$ EDF (583 ( \pm 120$)$ ) aunque no hubo diferencias significativas, el grupo de Jiu-Jitsu mostró valores más altos que el Grupo EDF.

\footnotetext{
${ }^{1}$ Universidade Federal do Ceará (UFC), Fortaleza - CE - Brasil. Docente no Instituto de Educação Física e Esportes. ORCID: <http://orcid.org/0000-0002-9267-0735>. E-mail: carlosas@ufc.br

${ }^{2}$ Universidade Federal do Ceará (UFC), Fortaleza - CE - Brasil. Laboratório de Biomecânica do Instituto de

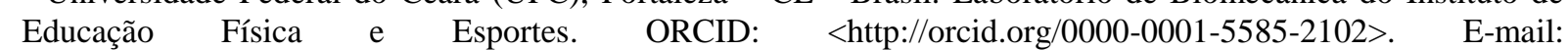
lincoln7777@hotmail.com

${ }^{3}$ Centro Universitário INTA (UNINTA), Sobral - CE - Brasil. Docente do Curso de Educação Física. ORCID: <http://orcid.org/0000-0002-5792-4429>. E-mail: stela.soares@uninta.edu.br
} 
Con los resultados obtenidos se puede concluir que la práctica del Jiu-Jitsu puede aportar beneficios para la salud como buenos o mejores que la práctica de la educación física.

PALABRAS CLAVE: Aptitud física. Salud. Educación física de la escuela. Jiu-Jitsu.

ABSTRACT: Jiu-Jitsu is a sport that has been growing a lot in recent years. The objective was to compare the levels of physical fitness among practicing and non-practitioners of jiu-jitsu students in a neighborhood in the city of Fortaleza/CE. The following variables were analyzed: BMI, flexibility, abdominal resistance and cardiorespiratory endurance, with 22 children, all males, divided into 2 groups with 11 individuals. It was observed that the Jiu-Jitsu group was better in all tests mainly in the flexibility tests (JJ: 31 ( \pm 2.00$), E D F: 28( \pm 4,07)$ ) and abdominal resistance (JJ: $30( \pm 4,42), E D F: 25( \pm 2.99))$, where it obtained a significant difference, already in the variables BMI (JJ: $20( \pm 2.02) E D F: 22( \pm 4,28)$ and cardiorespiratory Endurance (JJ: $654( \pm 127)$ EDF (583 ( \pm 120$))$ Although there was no significant difference, the Jiu-jitsu group showed higher values than the EDF group. With the results obtained it can be concluded that the practice of Jiu-jitsu can bring health benefits as good or better than the practice of physical education.

KEYWORDS: Physical fitness. Health. School physical education. Jiu-Jitsu.

\section{Introdução}

Segundo o MEC (2007) a educação é um direito de todo cidadão, sendo assim, o estado e a família têm como obrigação oferecer uma educação de qualidade (BRASIL, 1996). Ela abrange os processos formativos que se desenvolvem na vida familiar, na convivência humana e tem como finalidade o pleno desenvolvimento do educando. Os Parâmetros Curriculares Nacionais - PCNs (1998) se constituem como parâmetro de referência de qualidade para a educação em todo país e tem como função orientar e garantir a coerência dos investimentos no sistema educacional. Os PCNs têm como objetivos compreender a cidadania como participação social e política, assim como exercício de direitos e deveres políticos, civis e sociais no dia-adia; desenvolver o conhecimento ajustado de si mesmo e o sentimento de confiança em suas capacidades afetiva, física, cognitiva, ética, estética, de inter-relação pessoal e de inserção social (PCN, 1998).

De acordo com os Parâmetros Curriculares Nacionais (1998), a área de educação física é estabelecida como um conhecimento denominado de cultura corporal de movimento que trabalha o jogo, a lutas, a ginastica, o esporte, a dança, a capoeira e outras temáticas que tem interação com os principais problemas dessa cultura corporal de movimento e o contexto histórico-cultural dos alunos. 
Alves (2007) afirma que as escolas têm em média de uma a três aulas de educação física por semana esse número tende a cair principalmente quando se ingressa no ensino médio por conta da preocupação para entrar no ensino superior e do estresse com o vestibular. Porém, o autor ressalta que quando o aluno tem uma prática regular de atividade física ele consegue suportar o estresse desse período mais facilmente, mas com esse curto período de tempo para a prática fica muito difícil engajar alguma modalidade de forma eficiente. Em determinadas regiões do Brasil, como na região nordeste, especificamente no estado do Ceará, são determinadas pela Secretaria de Educação do Estado e da Prefeitura, duas aulas por semana, alternando entre teórica e prática, ou em muitas escolas, somente uma aula por semana.

Segundo Andrade e Devide (2006) alguns aspectos que podem dificultar o interesse e a prática da educação física está ligada a ambiente inadequado (quadra pequena ou inexistente); aulas muito repetitivas e desorganizadas; falta de habilidade e desprazer nos esportes oferecidos; brutalidade masculina; professores que não tem uma participação mais ativa na aula; desigualdade de habilidades por gênero; exclusão de indivíduos com menos habilidades; preferência da bola sempre para os meninos. Segundo Martinelli et al. (2006), o profissional de Educação Física muitas vezes contribui para o desinteresse dos alunos, pois os métodos utilizados para desenvolvimento das aulas, os conteúdos pouco relevantes, o relacionamento com os alunos, entre outros fatores, determina o participar ou não das aulas. Assim, fazendo com que as aulas de educação física não estejam alcançando seus objetivos, já que muitos problemas na prática são percebidos. Para tanto, avaliar torna-se fundamental, podendo contribuir trazendo informações pertinentes quanto à evolução dos alunos da rede escolar.

O principal objetivo da avaliação é o diagnóstico. Detectar as dificuldades da aprendizagem e suas causas, quando bem compreendido, possibilita grandes ganhos à educação e a aprendizagem do aluno se torna mais significativa. Na Educação Física a avaliação é, igualmente, um diagnóstico, portanto, deve ter o intuito de detectar possíveis falhas no processo ensino aprendizagem. Os aspectos cognitivos e afetivos devem fazer parte de uma avaliação que visualize o aluno na sua íntegra, porém, se faz necessário lembrar que estes dois aspectos, apesar de serem motivo de discussão de longa data, estão no início de uma longa caminhada. A avaliação que predomina nos meios escolares é a de domínio psicomotor. A aptidão física é sempre priorizada nas avaliações realizadas em Educação Física, e tal priorização se deve ao destaque dado, na graduação, às disciplinas práticas e à praticidade de sua realização (BRATIFISCHE, 2008).

Willrich, Azevedo e Fernandes (2009), afirmam que um aspecto muito importante para a vida futura das crianças é o motor. O desenvolvimento motor e um processo sequencial, 
continuo e relacionado à idade cronológica, em que o ser humano adquire uma enorme quantidade de habilidades motoras, que evoluem de movimentos simples e desorganizados para a execução de habilidades motoras altamente organizadas e complexas

Guedes (2007) em estudo realizado mostrou algumas diferenças motoras quando foram comparados alunos de um município brasileiro. Os testes apresentaram diferença entre os sexos, os rapazes levam vantagem no desempenho motor desde os sete anos, aumentando com a idade, claro que existem fatores biológicos que pode tem contribuído para essa vantagem dos rapazes. Da Silva (2010) realizou um estudo cujo objetivo era comparar as habilidades motoras de crianças desportistas e crianças que só praticavam a educação física escolar, no qual mostra que crianças que praticam somente a educação física escolar tiveram um desempenho bastante inferior quando comparado com crianças que faziam atividades esportivas. Da Silva (2010), ainda mostra que há outra diferença que sugere evolução alterada do desenvolvimento motor, que seria estudar em escola pública ou estudar em escola privada, sabendo que crianças de escola privada têm índices mais elevados do que crianças de escola pública. Fato importante, pelo visto, seria complementar as aulas de educação física escolar com atividades esportivas fora do horário da educação física escolar.

Diante dessa problemática, atividades como as lutas tornam-se um meio interessante, pois representa uma manifestação da cultura corporal (NASCIMENTO, 2014). Ávila (2017), diz que o jiu-jitsu dentro da educação (atividade) física escolar pode ser de grande ajuda para os professores com o intuito de diminuir a dificuldade que é falar das lutas na escola.

Segundo Rufino e Darido (2009), o fato que pode estar ligado ao jiu-jitsu não estar presente nas escolas públicas brasileiras como modalidade obrigatória nas aulas de educação física é o fato de muitas pessoas ainda verem a modalidade de forma distorcida, como se este esporte presente na escola fosse tornar os indivíduos mais violentos e agressivos. Reis, Suzuki e Gomes (2012), falam que, para os leigos, o jiu-jitsu não contribui para a formação do aluno diferente de outras lutas como, capoeira, judô e caratê, por isso existe certa restrição na área escolar. A Lei de Diretrizes e Bases da Educação (LDB) - Lei n 9.394/96 dar aos professores a possibilidade de explorar novos conteúdos para a disciplina além de novas metodologias da educação física escolar.

Na prática, poucas aulas de educação física são executadas nas escolas da cidade de Fortaleza. Contribuindo para isso, muitos professores são substitutos, não fazendo inserir o esporte como o professor efetivo na Escola em que leciona. $\mathrm{O}$ desenvolvimento na prática das habilidades motoras não são prioridades no planejamento dos mesmos. Falta de estrutura para a prática da educação física escolar, aulas sem motivação, tornando-se repetitivas, 
desinteressando os alunos. Sendo assim o jiu-jitsu pode surgir como uma alternativa para suprir essa falta de planejamento das escolas e auxiliar no desenvolvimento das crianças, pois a modalidade trabalha diversos aspectos como socialização, desenvolvimento motor, hierarquia e respeito. Sendo uma modalidade fácil de ser encontrada hoje em dia, com a popularização da modalidade não é difícil encontrar academias e projetos sociais nos mais diversos horários para atender as crianças e assim auxiliar na sua aprendizagem e desenvolvimento.

Dessa forma, resolveu-se realizar este estudo objetivando comparar os níveis de aptidão física entre escolares praticantes e não praticantes de Jiu-Jitsu.

\section{Metodologia}

O presente estudo foi do tipo descritivo de caráter exploratório, de cunho quantitativo e transversal. Segundo Gerhardt e Silveira (2009) o estudo descritivo é aquele que pretende descrever os fatos e fenômenos de determinada realidade. É caracterizado como Exploratório devido ao fato de a pesquisa proporcionar maior familiaridade com o problema, podendo verificar possíveis causas e discute-las.

Este estudo caracteriza-se por ser transversal, em que realizará somente uma coleta de dados através da aplicação de questionários, de cunho quantitativo, pois os resultados da pesquisa podem ser quantificados e recorre à linguagem matemática para descrever as causas de um fenômeno, as relações entre variáveis, etc. (GERHARDT; SILVEIRA, 2009).

Foram pesquisadas 22 pessoas, entre 7 a 16 anos proveniente de 2 populações. População A: 11 crianças que praticam atividade física na escola; População B: 11 crianças que praticam jiu-jitsu. Adotaram-se os seguintes critérios de inclusão na pesquisa: escolas na cidade de Fortaleza; crianças com idade de 7 a 14 anos; ambos os sexos; pelos menos 3 meses de pratica de educação física e 3 meses de prática de jiu-jitsu. Já como critério de exclusão foi estabelecido: o responsável pelo menor não assinar o TCLE. Assim, todos os responsáveis pelos sujeitos que participaram da pesquisa assinaram um Termo de Consentimento Livre e Esclarecido (TCLE). O estudo foi realizado seguindo todo o protocolo contido na Resolução n. ${ }^{\circ} 466 / 12$ do Conselho Nacional de Saúde (CNS), que estabelece as diretrizes e normas regulamentadoras de pesquisas envolvendo seres humanos.

Em um primeiro momento, houve o contato com os donos ou responsáveis pela academia de Jiu-Jitsu e pela escola onde foram coletados os dados do grupo II e I respectivamente, onde foram explicados os objetivos e detalhes do estudo. Após a autorização da administração da academia e da escola, foi encaminhado aos pais um termo solicitando a 
autorização de seus filhos como amostra da pesquisa, após todos os termos terem sido devidamente assinados os alunos devidamente matriculados em cada instituição foram convidados a participar do estudo. Após os contatos devidos, a pesquisa foi realizada baseada nos critérios de inclusão.

Após a assinatura, teve-se início a coleta, que foi dividia em 2 dias sendo 1 dia para cada grupo. A coleta foi realizada no horário normal da aula dos alunos. Nos dias de cada grupo foram realizados os testes referentes à aptidão física relacionada à saúde: massa corporal, estatura, IMC, flexibilidade e força de resistência abdominal e geral. Antes do início dos testes foi entregue a ficha de avaliação para os alunos, somente com seus dados pessoais preenchidos, os alunos entregaram a ficha ao professor ao realizar cada teste. O professor anotou o resultado do teste e devolve a ficha ao aluno.

Para caracterizar os participantes do estudo, foram mensuradas algumas variáveis, como: idade, gênero, massa corpórea e estatura. A idade, gênero, massa corpórea e estatura foram registrados em ficha específica de coleta de dados. Idade e gênero foram registrados em ficha específicas durante a coleta de dados.

Massa Corporal

- Instrumento: balança digital plenna com precisão de 100 gramas.

- Coleta de Dados: O sujeito avaliado se posicionou sobre a balança sem calçados e com o mínimo de roupa possível, a massa corporal e medida em Kg (GUEDES; GUEDES, 1995).

\section{Estatura}

- Instrumento: Fita métrica com no máximo 2metros

- Coleta de Dados: A fita métrica foi fixada na parede a 1 metro do solo e estendida de baixo para cima. Soma-se ao resultado medido na trena a distância á trena que é de 1 metro. $\mathrm{O}$ avaliado se posiciona junto a parede, sem calçados e a medida é tida do vértex a região plantar. (GUEDES; GUEDES, 1995).

Índice de Massa Corporal (IMC)

- Coleta de Dados: É determinado através do cálculo da razão entre a medida de massa corporal em quilogramas pela estatura em metros elevada ao quadrado. IMC $=$ Massa $(\mathrm{Kg}) /$ estatura (m)². (GUEDES; GUEDES, 1995). 
Tabela 01 - Pontos de corte, IMC

\begin{tabular}{c|c}
\hline IMC & Classificação \\
\hline Abaixo de 18,5 & Abaixo do peso \\
Entre 18,6 e 24,9 & Peso ideal \\
Entre 25,0 e 29,9 & Sobre peso \\
Entre 30,0 e 34,9 & Obesidade grau I \\
Entre 35,0 e 39,9 & Obesidade grau II (severa) \\
Acima de 40 & Obesidade III (Mórbida) \\
\hline
\end{tabular}

Fonte: autores

Flexibilidade (sentar-e-alcançar)

- Material: Banco de wells

- Coleta de Dados: Os alunos devem estar descalços. Sentam-se de frente do banco, com as pernas estendidas e unidas. Colocam uma das mãos sobre a outra e elevam os braços à vertical. Inclinam o corpo para frente e alcançam com as pontas dos dedos das mãos tão longe quanto possível sobre a régua graduada, sem flexionar os joelhos e sem utilizar movimentos de balanço (insistências). Cada aluno realizará duas tentativas. $\mathrm{O}$ avaliador permanece ao lado do aluno, mantendo-lhe os joelhos em extensão (GUEDES; GUEDES, 1995).

Para classificação do teste de sentar e alcançar foi utilizado Canadian Standardized Teste of Fitness (CSTF), utilizou-se os seguintes pontos.

Tabela 02 - Pontos de corte, Flexibilidade

\begin{tabular}{l|l|l|l|l|l|l}
\hline Idade & $\mathbf{1 5 - 1 9}$ & $\mathbf{2 0 - 2 9}$ & $\mathbf{3 0 - 3 9}$ & $\mathbf{4 0 - 4 9}$ & $\mathbf{5 0 - 5 9}$ & $\mathbf{6 0 - 6 9}$ \\
\hline Excelente & $>39$ & $>40$ & $>38$ & $>35$ & $>35$ & $>33$ \\
$\begin{array}{l}\text { Acima da } \\
\text { média }\end{array}$ & $34-38$ & $34-39$ & $33-37$ & $29-34$ & $28-34$ & $25-32$ \\
$\begin{array}{l}\text { Média } \\
\text { Abaixo da } \\
\text { média }\end{array}$ & $24-28$ & $25-29$ & $23-27$ & $18-23$ & $16-23$ & $15-19$ \\
\hline
\end{tabular}




\begin{tabular}{l|l|l|l|l|l|l}
\hline Ruim & $<23$ & $<24$ & $<22$ & $<17$ & $<15$ & $<14$ \\
\hline
\end{tabular}

Fonte: autores

Força-resistência (abdominal)

- Material: Colchonetes de ginástica e cronômetro.

- Coleta de Dados: O método utilizado foi o teste sit up's (repetições abdominais máximas em 1 minuto). $\mathrm{O}$ aluno posiciona-se em decúbito dorsal com os joelhos flexionados a 90 graus e com os braços cruzados sobre o tórax. O avaliador fixa os pés do estudante ao solo. Ao sinal o aluno inicia os movimentos de flexão do tronco até tocar com os cotovelos nas coxas, retornando a posição inicial (não é necessário tocar com a cabeça no colchonete a cada execução). $\mathrm{O}$ avaliador realiza a contagem em voz alta. $\mathrm{O}$ aluno deveria realizar o maior número de repetições completas em 1 minuto. O resultado e o número de repetições completos realizados em 1 minuto. (GUEDES; GUEDES, 1995).

Tabela 03 - Pontos de corte do teste de resistência abdominal

\begin{tabular}{r|r|l|l|l|r}
\hline \multicolumn{1}{l|}{ Idade } & Excelente & $\begin{array}{l}\text { Acima da } \\
\text { média }\end{array}$ & Média & $\begin{array}{l}\text { Abaixo da } \\
\text { média }\end{array}$ & \multicolumn{2}{l}{ Fraco } \\
\hline $\mathbf{1 5 - 1 9}$ & +48 & 42 a 47 & 38 a 41 & 33 a 37 & -32 \\
$\mathbf{2 0 - 2 9}$ & +43 & 37 a 42 & 33 a 36 & 29 a 32 & -28 \\
$\mathbf{3 0 - 3 9}$ & +36 & 31 a 35 & 27 a 30 & 22 a 26 & -21 \\
$\mathbf{4 0 - 4 9}$ & +31 & 26 a 30 & 22 a 25 & 17 a 21 & -16 \\
$\mathbf{5 0 - 5 9}$ & +26 & 22 a 25 & 18 a 21 & 13 a 17 & -12 \\
$\mathbf{6 0 - 6 9}$ & +23 & 17 a 22 & 12 a 26 & 07 a 11 & -06 \\
\hline
\end{tabular}

Fonte: autores

\section{Resistência Geral (6 minutos)}

- Material: Local plano, cronômetro e ficha de registro. Material numerado para fixar às costas dos alunos identificando-os claramente para que o avaliador possa realizar o controle do número de voltas. Trena métrica.

- Coleta de Dados: Divide-se os alunos em grupos adequados às dimensões da pista. Observa-se a numeração dos alunos na organização dos grupos, facilitando assim o registro dos anotadores. Tratando-se de estudantes com cabelos longos, observa-se o comprimento dos cabelos para assegurar que o número às costas fique visível. Informase aos alunos sobre a execução dos testes dando ênfase ao fato de que devem correr o 
maior tempo possível, evitando piques de velocidade intercalados por longas caminhadas. Informa-se que os alunos não deverão parar ao longo do trajeto e que se trata de um teste de corrida, embora possam caminhar eventualmente quando sentiremse cansados. Durante o teste, informa-se ao aluno a passagem do tempo 2, 4 e 5 (“Atenção: falta 1 minuto). Ao final do teste soará um sinal (apito) sendo que os alunos deverão interromper a corrida, permanecendo no lugar onde estavam (no momento do apito) até ser anotada ou sinalizada a distância percorrida. Todos os dados serão anotados em fichas próprias devendo estar identificado cada aluno de forma inequívoca (GUEDES; GUEDES, 1995).

Tabela 04 - Pontos de corte de resistência geral.

\begin{tabular}{rl|l|l|l|l|l}
\hline Idade & Fraco & Razoável & Bom & M. Bom & Excelente \\
\hline $\mathbf{7}$ & $>735$ & $735-785$ & $786-824$ & $825-923$ & $>=924$ \\
$\mathbf{8}$ & $<773$ & $773-825$ & $826-878$ & $879-1009$ & $>=1010$ \\
$\mathbf{9}$ & $<845$ & $845-899$ & $900-965$ & $966-1096$ & $>=1097$ \\
$\mathbf{1 0}$ & $<880$ & $880-941$ & $942-1009$ & $1010-1157$ & $>=1158$ \\
$\mathbf{1 1}$ & $<915$ & $915-977$ & $978-1049$ & $1050-1189$ & $>=1190$ \\
$\mathbf{1 2}$ & $<965$ & $965-1029$ & $1030-1109$ & $1100-1254$ & $>=1255$ \\
$\mathbf{1 3}$ & $<983$ & $983-1082$ & $1083-1158$ & $1159-1319$ & $>=1320$ \\
$\mathbf{1 4}$ & $<1068$ & $1068-1134$ & $1135-1209$ & $1210-1371$ & $>=1372$ \\
$\mathbf{1 5}$ & $<1120$ & $1120-1186$ & $1187-1261$ & $1262-1434$ & $>=1435$ \\
$\mathbf{1 6}$ & $<1150$ & $1150-1219$ & $1220-1288$ & $1289-1504$ & $>=1505$ \\
$\mathbf{1 7}$ & $<1156$ & $1156-1219$ & $1220-1288$ & $1289-1504$ & $>=1505$ \\
\hline
\end{tabular}

Fonte: autores

\section{Análise dos resultados}

Os resultados do projeto foram analisados estatisticamente através de alternativas estatísticas sugeridas para pesquisa avaliativa no qual o tratamento dos dados é feito através de estatísticas descritivas (Média \pm DP), não exigindo, portanto, aleatoriedade da amostra, distribuição probabilística, delimitação da dimensão da amostra e os pressupostos da estatística inferencial. Sendo assim, para estimar os níveis das capacidades físicas de crianças praticantes 
e não praticantes de atividades desportivas, todas as análises das estatísticas foram realizadas no programa SPSS for Windows 18.0.

\section{Resultados}

Participaram do estudo 22 sujeitos, divididos em 2 grupos de 11 com média de idade de 13 anos para ambos os grupos, todos do gênero masculino. Um grupo que treinou Jiu-jitsu e outro que fez educação física escolar, os indivíduos do grupo Jiu-jitsu se mostraram mais leves com uma media de massa corporal de $(51,272 \pm 8,66)$ em relação os indivíduos do grupo da educação física com média de 56,54 ( $\pm 13,4)$, porém o grupo da educação física era minimamente mais alto que o grupo Jiu-jitsu, o grupo jiu-jitsu obteve média de 1,58 $\pm 0,10$ enquanto o grupo EDF obteve 1,59 $\pm 0,10$ de altura, obtendo $1 \mathrm{~cm}$ de vantagem para o grupo Jiu-jitsu.

Tabela 05 - Características dos Sujeitos do Estudo

\begin{tabular}{c|l|l|l}
\hline & \multicolumn{1}{|c|}{$\begin{array}{r}\text { Grupo Jiu-jitsu } \\
\mathbf{n = 1 1}\end{array}$} & \multicolumn{1}{|c|}{$\begin{array}{c}\text { Grupo EDF } \\
\mathbf{n = 1 1}\end{array}$} & \\
\hline Idade (Anos) & $13 \pm, 90$ & $13 \pm 1,02$ &, 828 \\
Gênero (M/F) & $\mathrm{M}$ & $\mathrm{M}$ & \\
Massa Corporal (Kg) & $51,272 \pm 8,66$ & $56,54 \pm 13,43$ &, 287 \\
Estatura (m) & $1,58 \pm, 10$ & $1,59 \pm, 10$ &, 772 \\
\hline
\end{tabular}

Fonte: Pesquisa direta, 2019.

\section{Apresentação dos resultados do Índice de Massa Corporal (IMC)}

Os sujeitos do grupo jiu-jitsu obtiveram valores mais baixos de IMC em ralação ao grupo EDF, o grupo jiu-jitsu obteve média de $20 \pm 2,02$ de IMC enquanto o grupo EDF $22 \pm$ 4,28. Na variável flexibilidade o grupo jiu-jitsu teve valores mais altos que o grupo EDF, com $31 \mathrm{~cm}( \pm 2,00)$ para o grupo jiu-jitsu e $28 \mathrm{~cm}( \pm 4,07)$ para o grupo EDF, logo dando uma diferença significativa entre os dois grupos. Na variável de resistência abdominal mais uma vez houve diferença significativa entre os grupos onde o grupo jiu-jitsu obteve uma média de 30 $\operatorname{Rep} / \min ( \pm 4,42)$ enquanto o grupo EDF $25 \mathrm{Rep} / \min ( \pm 2,99)$. E na ultima variável analisada que foi a resistência cardiorrespiratória o grupo jiu-jitsu obteve uma média superior ao grupo 
EDF, a média do grupo jiu-jitsu foi de $654 \mathrm{~m}( \pm 127,15)$ enquanto do grupo EDF foi de $583 \mathrm{~m}$ $( \pm 120,27)$.

Tabela 06 - Valores Médios das Variáveis Analisadas, por Grupo.

\begin{tabular}{|c|c|c|c|}
\hline & Grupo Jiu-Jitsu & Grupo EDF & $\begin{array}{l}\text { Valor } \\
\mathbf{P}\end{array}$ \\
\hline IMC (kg/m²) & $20 \pm 2,02$ & $22 \pm 4,28$ & 0,230 \\
\hline Flexibilidade (cm) & $31 \pm 2,00$ & $28 \pm 4,07$ & $0,013^{*}$ \\
\hline Abdominal (rep/Mim) & $30 \pm 4,42$ & $25 \pm 2,99$ & $0,006^{*}$ \\
\hline Resistencia Cardio (m) & $654 \pm 127,15$ & $\begin{array}{r}583 \pm \\
120,27\end{array}$ & 0,194 \\
\hline
\end{tabular}

Fonte: Pesquisa direta, 2019 - *= diferença significativa

A variável IMC do grupo jiu-jitsu, obteve uma média de $20 \mathrm{~kg} / \mathrm{m}^{2}$, já o Grupo EDF obteve uma media de $22 \mathrm{~kg} / \mathrm{m}^{2}$, onde 5 indivíduos, equivalente a $46 \%$ do grupo jiu-jitsu estão abaixo do peso ideal e os outros 6(54\%) estão dentro da margem de peso ideal, agora o grupo EDF se encontram 3 indivíduos (28\%) que estão a baixo do peso enquanto 6(54\%) estão no peso ideal, porém 1 criança (9\%) está acima do peso, e outro individuo (9\%) está com obesidade grau I.

O grupo de educação física mostrou seu índice de massa corporal (IMC) levemente mais elevado $(22 \pm 4,28)$ quando comparado ao grupo jiu-jitsu $(20 \pm 2,02)$, onde no grupo EDF cerca de $18 \%$ do grupo está acima do peso ideal para a faixa etária, porém ambos estão com suas médias dentro do peso ideal.

Tabela 07 - Resultado Médio da Variável Índice de Massa Corporal (IMC).

\begin{tabular}{l|c|c}
\hline & Grupo Jiu-jitsu & Grupo EDF \\
\hline Abaixo do peso & $5(46 \%)$ & $3(28 \%)$ \\
Peso ideal & $6(54 \%)$ & $6(54 \%)$ \\
Sobrepeso & 0 & $1(9 \%)$ \\
Obesidade grau l & 0 & $1(9 \%)$ \\
Obesidade grau ll & 0 & 0 \\
\hline
\end{tabular}

Fonte: Pesquisa direta, 2019. 


\section{Apresentação dos resultados do nível de flexibilidade}

O grupo jiu-jitsu teve uma media de $31 \pm 2,00 \mathrm{~cm}$ enquanto grupo EDF demonstrou uma media de $28 \pm 4,07 \mathrm{~cm}$ no teste de flexibilidade, onde no grupo jiu-jitsu 3 crianças, equivalente a $28 \%$ do grupo demonstraram níveis dentro da media enquanto no grupo EDF somente 1 individuo (9\%) mostrou valores que se encaixassem na média, $63 \%$ do grupo jiu-jitsu e 45,5\% do grupo EDF demonstraram estar a baixo do média e somente 1 criança do jiu-jitsu demonstrou ter níveis ruins de flexibilidade, já no Grupo EDF 5 crianças equivalente a 45,5\% demonstraram ter níveis tão ruins de flexibilidade.

As crianças do grupo jiu-jitsu mostraram níveis de flexibilidade superiores $31 \mathrm{~cm} \pm 2,00$ quando comparado ao grupo EDF $28 \pm 4,07$, onde a média do grupo jiu-jitsu se encaixaria dentro da média segundo a classificação da tabela 02, enquanto o Grupo EDF obteve valores abaixo da média segundo o ponto de corte da tabela, apesar dos valores próximos a media do grupo jiu-jitsu se mostrou mais elevada, com isso dando uma diferença significativa entre os grupos.

Tabela 08 - Resultado Médio da Variável Flexibilidade

\begin{tabular}{l|c|c}
\hline \multicolumn{2}{c}{ Grupo Jiu-jitsu } & Grupo EDF \\
\hline Excelente & 0 & 0 \\
Acima da media & 0 & 0 \\
Média & $3(28 \%)$ & $1(9 \%)$ \\
Abaixo da média & $7(63 \%)$ & $5(45.5 \%)$ \\
Ruim & $1(9 \%)$ & $5(45.5 \%)$ \\
\hline
\end{tabular}

Fonte: autores

\section{Apresentação dos resultados da resistência abdominal (força) dos sujeitos}

Todos as 11 crianças ou seja 100\% do grupo EDF demonstrou níveis ruins de força abdominal, enquanto 7 crianças $(64 \%)$ do grupo jiu-jitsu mostrou que também tem valores ruins, $27 \%$ e $9 \%$ do grupo jiu-jitsu demonstraram níveis abaixo da média e dentro da media, respectivamente.

Novamente o grupo que pratica jiu-jitsu se mostrou com um maior nível de resistência abdominal, com média de 30,81( $\pm 4,42)$ enquanto o grupo EDF obteve uma média de $35,81( \pm 2,99)$, com isso levando novamente a uma diferença significativa entre os grupos. 
Tabela 09 - Resultado Médio da Variável resistência abdominal

\begin{tabular}{l|c|c}
\hline \multicolumn{2}{c}{ Grupo Jiu-jitsu } & Grupo EDF \\
\hline Excelente & 0 & 0 \\
Acima da media & 0 & 0 \\
Média & $1(9 \%)$ & 0 \\
Abaixo da média & $3(27 \%)$ & 0 \\
Ruim & $7(64 \%)$ & $11(100 \%)$ \\
\hline
\end{tabular}

Fonte: Pesquisa direta, 2019.

\section{Apresentação dos resultados da resistência cardiorrespiratória dos sujeitos}

Na variável de resistência cardiorrespiratória 6(54\%) dos indivíduos do grupo jiu-jitsu e 8(73\%) do grupo EDF demonstraram um desempenho fraco no teste de resistência de 6 minutos, enquanto $3(27 \%)$ de ambos os grupos demonstraram um desempenho razoável e 2(19\%) do grupo jiu-jitsu demonstrou um desempenho bom no teste, nenhum dos grupos consegui alcançar maiores resultados.

Nessa variável apesar dos valores entre os grupos serem levemente mais favoráveis para o grupo jiu-jitsu com média de 654,54( $(127,15)$, enquanto a média do grupo EDF foi de $583,63( \pm 120,27)$, não foi o suficiente para obter uma diferença significativa entre os grupos.

Tabela 10 - Resultado Médio da Variável Resistencia cardiorrespiratória

\begin{tabular}{l|c|c}
\hline \multicolumn{2}{c}{ Grupo Jiu-jitsu } & Grupo EDF \\
\hline Excelente & 0 & 0 \\
Muito bom & 0 & 0 \\
Bom & $2(19 \%)$ & 0 \\
Razoável & $3(27 \%)$ & $3(27 \%)$ \\
Fraco & $6(54 \%)$ & $8(73 \%)$ \\
\hline
\end{tabular}

Fonte: Pesquisa direta, 2019.

\section{Discussão}

\section{Índice de Massa Corporal dos Sujeitos}

De acordo com a tabela 07, o grupo jiu-jitsu se encontra com o IMC dentro do peso ideal, com média de $20 \pm 2,02$, grupo EDF obteve uma média de $22 \pm 4,28$ que também o coloca na classificação de peso ideal, porém teve $18 \%$ dos seus indivíduos sobrepeso ou acima do peso. 
Em estudo realizado por Burgos et al., (2012), com crianças de 7 a 17 anos escolares, em um município do Rio Grande Do sul, analisou os níveis de aptidões físicas relacionados a saúde, onde as crianças de 13 anos obtiveram uma média de IMC de 19,78ะ3,56, classificandoos como peso ideal. O nosso estudo teve resultado parecido, onde a média do grupo jiu-jitsu foi de $(20 \pm 2,02)$ enquanto do grupo EDF foi $(22 \pm 4,28)$, Valores próximos, onde tanto os grupos do nosso estudo como o grupo do estudo de Burgos se encontram em uma classificação de peso ideal

Bergman et al., (2005) em seu estudo no estado do Rio Grande do Sul, cujo objetivo era avaliar variáveis da aptidão física relacionadas a saúde em crianças e adolescentes escolares, onde as crianças da faixa etária de 13 anos obtiveram uma média de IMC 19,81( $\pm 3,53)$ valores que indicam a média dentro do peso ideal, média próxima a do grupo jiu-jitsu com $20( \pm 2,02)$.

Ulbrich et al., (2007) realizou um estudo sobre aptidão física em crianças e adolescentes com diferentes estágios maturacionais que praticam regularmente alguma atividade esportiva de 2 a 5 vezes por semana, onde as crianças com a média de idade de 13 anos apresentaram uma média de 20,58 $( \pm 3,39)$ media considera dentro do peso ideal segundo a tabela de IMC, resultados próximos aos obtidos pelo grupo jiu-jitsu $(20 \pm 2,02)$.

Santos et al., (2010) realizou um estudo cujo objetivo era comparar crianças e adolescentes brasileiros de escolar publicas e privadas com curvas de referência para crescimento físico, a média de IMC de crianças com a faixa etária de 13 anos foi de 19,4( $\pm 3,2)$ valor dentro do peso ideal para a faixa etária e valor próximo ao obtido pelos grupos EDF (22 $\pm 4,28)$ e jiu-jitsu $(20 \pm 2,02)$.

Hobold (2003) realizou um estudo de aptidão física relacionada a saúde no município de Marechal Cândido Rondon, Paraná, com crianças e adolescentes do nível fundamental e médio da faixa etária de 7 a 17 anos, onde uma das variáveis estudada foi o IMC, onde as crianças com idade de 13 anos obtiveram uma média 18,4( $\pm 2,9)$ valor que os coloca um pouco abaixo do peso, segundo a tabela de classificação.

Quando comparados os estudos citados com o trabalho em questão, as crianças do grupo jiu-jitsu estivam dentro da média dos estudos, já o grupo EDF apesar de ter também uma classificação dentro do peso ideal, sua média foi levemente maior. Isso pode ter ocorrido, talvez, por conta de seus estados maturacionais, ou por conta de sua genética, ou ainda por conta do ritmo das aulas de educação física escolar que não tem uma intensidade considerável.

\section{Nível de flexibilidade dos sujeitos}


Como citado anteriormente na tabela 08 o grupo jiu-jitsu teve uma média de $31 \mathrm{~cm}( \pm$ $2,00)$, três centímetro amais do que o grupo EDF com média de $28 \mathrm{~cm}( \pm 4,07)$, onde o grupo jiu-jitsu teve valores mais elevados do que o grupo EDF, porém ambos tem valores dentro da média, mesmo a grande maioria do resultados obtidos pelo grupo EDF se concentraram em ruim ou abaixo da média, $91 \%$ do grupo teve resultado abaixo da média.

Um estudo realizado por Rassilan e Guerra (2006) em um município de Minas Gerais avaliou a flexibilidade em crianças entre 7 a 14 anos escolares, onde a média da variável na faixar etária de 13 anos em meninos foi de 20,3( $\pm 8,1)$, valores considerados ruins segundo a tabela de classificação e bem inferiores quando comparados aos grupos do nosso estudo.

Minatto, et al. (2010) realizaram um estudo com escolares de 8 a 17 anos, onde os níveis de flexibilidade de crianças de 13 anos foi de 24,9 ( \pm 7$)$, valores considerados abaixo da média, quando comparado ao nosso estudo onde os grupo jiu-jitsu obteve uma média de 31 e o grupo EDF $28 \mathrm{~cm}$, o estudo de Minatto apresenta valores bem inferiores.

Bergman et al., (2005) realizaram um estudo no estado do Rio Grande do Sul analisando a aptidão física relacionada a saúde das crianças e adolescentes, onde os níveis de flexibilidade obtidos em crianças da mesma faixa etária de 13 anos foi de 20,37 $( \pm 9,08)$, valor considero ruim do nível de flexibilidade.

Ulbrich et al. (2007) realizaram um estudo de aptidão física em crianças de diferentes estados maturacionais, onde as crianças do estágio 4 que tem a media de idade similar ao publico do nosso estudo 13,7 anos, onde as crianças desse estagio apresentaram um nível de flexibilidade de 22,68 $( \pm 8,0) \mathrm{cm}$, também níveis considerados ruins na tabela de classificação de flexibilidade.

Burgos et al., (2012) realizaram um estudo com crianças escolares de 7 a 17 anos avaliando seus níveis de aptidão física onde uma das variáveis analisadas foi a flexibilidade, as crianças da mesma faixa etária do o nosso estudo, 13 anos, obtiveram resultados com 20,64 $( \pm 7,0) \mathrm{cm}$, que tem níveis de flexibilidade considerados ruins segundo a tabela.

Em todos os artigos citados no tópico apresentaram valores bem inferiores quando comparados aos grupos do nosso trabalho, um provável motivo seja o numero de crianças em cada estudo onde todos os citados tiveram números bastante elevados enquanto o trabalho só tem 22 crianças divididas em dois grupos de 11, outro provável motivo pode ser que as crianças da EDF já estão bastante tempo praticando, enquanto crianças do jiu-jitsu apresentaram valores mais elevados por conta da especificidade do esporte que requer muita flexibilidade em dados momentos do combate, pelo grande requerimento da flexibilidade no jiu-jitsu, obteve-se uma diferença significativa com relação ao grupo EDF. 


\section{Resistência abdominal (força) dos sujeitos}

De acordo com os dados da tabela 09,100\% do grupo EDF obteve um resultado ruim no teste de abdominal com uma média de $25( \pm 2,99)$, enquanto o grupo jiu-jitsu com uma média de $30( \pm 4,42)$, obteve resultados mais heterogêneos mesmo os resultados em sua maioria sendo abaixo da media, são mais elevados quando comparados ao grupo EDF.

Burgos et al., (2012) realizou um estudo em crianças escolares com idades variando entre 7 e 17 anos onde nesse estudo ele avaliou a capacidade da resistência abdominal dos sujeitos, as crianças com a mesma média de idade do nosso trabalho, 13 anos, obtiveram uma media de 31,15 $\pm 9,25 \mathrm{rep} / \mathrm{min}$, resultado considerado fraco pela tabela dos pontos de corte, valores próximos a média do grupo jiu-jitsu $30( \pm 4,42)$ e valores bem superiores a média do grupo EDF $25( \pm 2,99)$.

Segundo o estudo citado anteriormente de Ulbrich et al. (2007) uma das variáveis analisada foi o teste de resistência abdominal, onde as crianças de 13 anos apresentaram uma

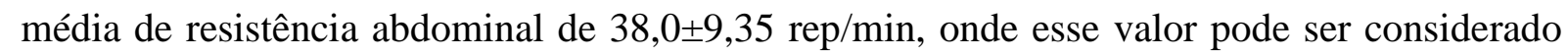
dentro da média, segundo a tabela, valor bem mais alto quando comparado as médias dos grupos do nosso estudo.

Segundo o estudo de Bergman et al., (2005), uma das variáveis analisada foi o teste de resistência abdominal, onde a média de abdominais por minuto das crianças com a faixa etária

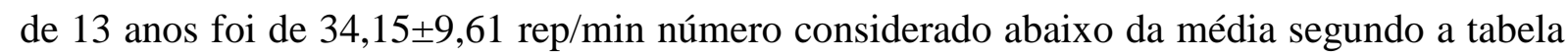
de classificação, um pouco mais elevado do que a média das crianças que praticavam jiu-jitsu $30( \pm 4,42)$ e bem maior quando comparado ao grupo EDF $25( \pm 2,99)$.

Segundo o estudo de Borges (2009) realizado em um município do Paraná com crianças da faixa etária de 7 a 14 anos, cujo objetivo era verificar seu desempenho, uma das avariáveis analisadas foi a de resistência abdominal em 1 minuto onde as crianças da faixa etária de 13

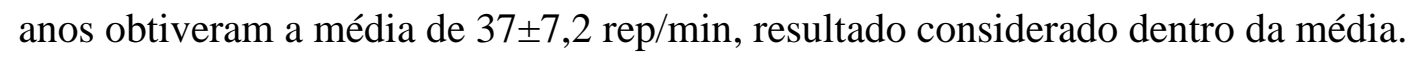

Guedes e Guedes (1995) realizou um estudo no município de Londrina-PR com o intuito de avaliar as capacidades físicas relacionadas a saúde em crianças e adolescentes de escolas publicas e particulares com idade entre 7 e 17 anos, onde o grupo entre 11-14 anos apresentou uma média de 31,5 rep/min, valor considerado fraco, um valor bem aproximado a média do grupo jiu-jitsu $30( \pm 4,42)$ porem elevado em relação ao grupo do grupo EDF $25( \pm 2,99)$.

Os valores dos estudos mostraram valores mais elevados quando comparados a os do nosso estudo, mesmo o grupo jiu-jitsu tento valores mais aproximados por conta que se tem uma quantidade maior de exercícios abdominais nas suas aulas quando comparado ao grupo 
EDF, os resultados podem ter destoado além do numero de participantes o perfil das crianças por região pode ter interferido nos resultados, porém como a intensidade das aulas de EDF não é tão elevada pode ter contribuído para a baixa média, além de não ter tantos exercícios abdominais nas aulas de educação física como no jiu-jitsu pode ter influenciado para obter uma diferença significativa entre os grupos.

\section{Resistência cardiorrespiratória dos sujeitos}

De acordo com a tabela 10 os indivíduos do grupo EDF apresentaram um rendimento de fraco e razoável no teste de resistência de 6 minutos, enquanto o grupo jiu-jitsu demonstrou números levemente superiores sendo $19 \%$ do grupo tendo um resultado considerado bom para os níveis da idade, enquanto a média do grupo EDF foi $583( \pm 120,27)$ a do grupo jiu-jitsu foi levemente superior com $654( \pm 127,15)$, porém ambas as médias dos grupos demonstram resultados fracos, segundo a tabela de classificação.

Lorenzi (2006) realizou um estudo com crianças de 10 a 14 anos utilizando o teste de corrida de 6 e 9 minutos, onde no teste de 6 minutos os indivíduos obtiveram uma média de 996,4 $( \pm 179,1)$ resultado considerado razoável, porém valores muito superiores quando comparados aos grupo jiu-jitsu $654( \pm 127,15)$ e ao grupo EDF $583( \pm 120,27)$.

Segundo o estudo de Bergman et al., (2005) citado anteriormente, uma das variáveis analisadas no estudo foi a corrida de 9 minuto onde as crianças obtiveram uma média 1442,31 $( \pm 288,04)$ que pode ser considerado razoável, porém ótimos resultados quando comparado as fracas medias dos dois grupos do nosso estudo.

Gaya (2002) realizou um estudo de aptidão física relacionada à saúde com crianças escolares de 7 a 17 anos escolares em toda a região sul, e uma das variáveis analisadas foi o teste de caminhada de 9 minutos, onde a média obtida pelo grupo da faixa etária de 13 anos foi de 1470,12 ( \pm 270,93) um resultado considerado razoável, com valores bem superiores em relação ao grupo jiu-jitsu e o grupo EDF.

Luguetti, Ré e Böhme (2010) em estudo realizado na região centro-oeste da cidade de São Paulo, para descobrir os níveis de aptidão física com crianças escolares de 7 a 16 anos, onde foi analisado o teste de caminhada de 9 minutos, e foi obtida uma media de $1400( \pm 194)$, resultado considerado fraco para a faixa etária de 13 anos, mesma classificação pressente nos grupos jiu-jitsu e EDF. 
Andreasi et al., (2010) realizaram um estudo sobre aptidão física com crianças da faixa etária de 7 a 15 anos em um munícipio do estado de São Paulo que cursavam o ensino fundamental $\left(2^{\circ}\right.$ ao $9^{\circ}$ ano), onde foram analisadas algumas variáveis, dentre elas o teste de correr/andar de 9 minutos, onde foi obtido uma média de 1271,1 $( \pm 221,4)$ um valor considerado fraco para a faixa etária.

Uma parte dos estudos obtiveram valores fracos com relação aos valores da tabela, mesmo esses valores sendo bastante superiores os dos grupos jiu-jitsu e EDF, mesmo o grupo jiu-jitsu tendo indivíduos que conseguiram desempenhos considerados bons, a média do grupo se manteve fraca, assim como o grupo EDF, quando comparado o grupo jiu-jitsu ao grupo EDF, o grupo jiu-jitsu obteve melhores valores, fator que pode ser explicado por conta da especificidade do esporte que requer mais intensidade cardiorrespiratória e pelo tempo de pratica de alguns participantes, já no grupo EDF o conteúdo das aulas pode ter influenciado no resultado, pois as atividades planejadas não são de uma intensidade elevada e com exercícios relativamente leves para o trabalho cardiorrespiratório, e mesmo com uma média considerada ruim o grupo jiu-jitsu tem um trabalho bem mais elevado na parte cardiorrespiratória por conta da dinâmica da modalidade.

\section{Considerações finais}

O jiu-jitsu tem bastante a contribuir no desenvolvimento das crianças não somente nas variáveis analisadas nesse estudo, mas como também diferentes habilidades corporais e cognitivas, pois as crianças precisam realizar diversos movimentos com o corpo, memória, atenção, percepção, relacionamento interpessoal entre outras competências.

De acordo com os resultados do nosso estudo, as variáveis analisadas mostraram que o grupo jiu-jitsu obteve médias superiores quando comparado ao grupo EDF.

Na variável IMC ambos os grupos estiveram dentro da média dos outros estudos, porém o grupo jiu-jitsu $(20 \pm 2,02)$ obteve um valor mais baixo de peso ideal, enquanto o grupo EDF $(22 \pm 4,28)$, que apesar de também estar no peso ideal está mais próximo do sobrepeso, com isso o grupo jiu-jitsu tem melhores índices de massa corporal, porém ambos estão no peso ideal.

Nos testes de flexibilidade ambos os grupos se demonstraram bem superiores aos estudos comparativos, porém mais uma vez o grupo jiu-jitsu com média de $(31 \pm 2,00)$ leva vantagem em relação ao grupo $\mathrm{EDF}(28 \pm 4,07)$, uma diferença de $3 \mathrm{~cm}$ na média, dando uma diferença significativa entre os grupos. 
No teste de abdominais Rep/min os estudos com quais foram comparados, os grupos jiu-jitsu e EDF ficaram a baixo da media dos mesmos, mesmo o grupo jiu-jitsu chegando próximo da média de alguns estudos, mas quando comparados entre si o grupo jiu-jitsu com uma média de $(30 \pm 4,42)$, leva vantagem com um valor bem mais elevado que o grupo EDF $(25 \pm 2,99)$, mais uma vez dando diferença significativa entre os grupo com uma vantagem media de 5 reps.

$\mathrm{Na}$ última variável analisada, que foi resistência cardiorrespiratória, os grupos obtiveram resultados considerados fracos, com uma média de $(654 \pm 127,15)$ para o grupo jiujitsu e de $(583 \pm 120,27)$ para o grupo EDF, enquanto os estudos comparativos obtiveram resultados dentro da média, mesmo com resultados fracos, também nessa variável o grupo jiujitsu se mostrou superior ao grupo EDF.

Em geral pode-se considerar que os objetivos foram alcançados, pois o objetivo era comparar as variáveis e mostrar que a pratica do jiu-jitsu pode trazer benefícios a saúde tanto quanto a educação física, e apresentou-se que a pratica da modalidade pode trazer mais benéficos que a educação física, ficou claro com os resultados mostrados que o grupo jiu-jitsu se saiu superior em todas as variáveis analisadas, então o jiu-jitsu pode trazer benefícios ao desempenho físico relacionado a saúde, ate mais que a própria educação física.

\section{REFERÊNCIAS}

ALVES, U. S. Não ao sedentarismo, sim à saúde: contribuições da Educação Física escolar e dos esportes. O Mundo da Saúde, São Paulo: out/dez, 2007.

ANDRADE, E. B; DEVIDE, F. Auto-exclusão nas aulas de educação física escolar: representações de alunas do Ensino Médio sob enfoque de gênero. FIEP Bulletin, Foz do Iguaçu, v. 76, p. 318-321, 2006. Special edition.

ANDREASI, V.; MICHELIN, E.; RINALDI, A. E. M.; BURINI, R. C. Aptidão física associada às medidas antropométricas de escolares do ensino fundamental. Jornal de Pediatria, 497-502, 2010.

ÁVILA, D. X. de. Reflexões sobre o jiu-jítsu brasileiro como conteúdo da educação física nas fases iniciais do ensino fundamental. Tese de Graduação, Universidade Federal de Santa Catarina - Centro de Desportos - Educação Física - Licenciatura. Santa Catarina, 2017.

BERGMAN, G. G.; LORENZI, T. D. C.; GARLIPP, D. C.; MARQUES, A. C.; ARAÚJO, M.; MACHADO, D.; LEMOS, A. T. Aptidão física relacionada à saúde de crianças e adolescentes do Estado do Rio Grande do Sul. Revista Perfil. Porto Alegre, vol. 7, n. 7 (2005), p. 12-21, 2005. 
BORGES, G. A., Avaliação do desempenho motor de crianças e adolescentes de 7 a 14 anos de Marechal Cândido Rondon, Paraná. Lecturas Educación Física y Deportes, v.123, 2009.

BRASIL. Lei de Diretrizes e Bases da Educação - LDB 9394/96. Disponível em: www.planalto.gov.br/ccivil_03/leis/L9394.htm. Acesso em 13 nov., 2018.

BRASIL. Ministério da Educação e do Desporto, Secretaria de Educação Fundamental. Parâmetros curriculares nacionais. Brasília: MEC/SEF, 1998.

BRASIL. MEC/SEF. Parâmetros Curriculares Nacionais - Ensino fundamental. Brasília/DF: MEC/SEF, 2007.

BRATIFISCHE, S. A. Avaliação em educação física: um desafio. Journal of Physical Education, v. 14, n. 2, p. 21-31, 2008.

BURGOS, M. S.; REUTER, C. P.; TORNQUIST, L.; PICCIN, A. S.; RECKZIEGEL, M. B.; POHL, H. H.; BURGOS, L. T. Perfil de aptidão física relacionada à saúde de crianças e adolescentes de 7 a 17 anos. Journal of the Health Sciences Institute, 30 (2), 171-175, 2012.

DA SILVA, C. M. M. Diferenças motoras entre crianças desportistas e crianças somente praticantes de Educação Física escolar. Revista Espaço Acadêmico, v. 9, n. 105, p. 78-86, 2010.

GAYA, A. C. A. Aptidão física relacionada à saúde: um estudo piloto sobre o perfil de escolares de 7 a 17 anos na região Sul do Brasil. Revista Perfil. Porto Alegre. Vol. 6, n. 6 (2002), p. 50-60, 2002.

GERHARDT, T. E.; SILVEIRA, D. T. Métodos de pesquisa. Plageder, 2009.

GUEDES, D. P. Implicações associadas ao acompanhamento do desempenho motor de crianças e adolescentes. Revista Brasileira de Educação Física e Esporte, v. 21, n. esp, p. 37-60, 2007.

GUEDES, D. P.; GUEDES, J. E. R. P. Aptidão física relacionada à saúde de crianças e adolescentes: avaliação referenciada por critério. Revista Brasileira de Atividade Física \& Saúde, 1(2), 27-38, 1995.

HOBOLD, E. Indicadores de aptidão física relacionada a saúde de crianças e adolescentes do município de Marechal Cândido Rondon - Paraná- Brasil. Tese (Mestrado em atividade física relacionada a saúde) - Faculdade de Educação Física, Universidade Federal de Santa Catarina, 2003.

LORENZI, D, C, L. Testes de corrida/caminhada de 6 e 9 minutos: validação e determinantes metabólicos em crianças e adolescentes. Tese (Mestrado em ciência do movimento humano) - Faculdade de educação física, Universidade Federal do Rio Grande do Sul, 2006. 
LUGUETTI, C. N.; RÉ, A. H. N.; BÖHME, M. T. S. Indicadores de aptidão física de escolares da região centro-oeste da cidade de São Paulo. Rev bras cineantropom desempenho hum, 12(5), 331-7, 2010.

MARTINELLI, C. R. et al. Educação Física no Ensino Médio: motivos que levam as alunas a não gostarem de participar das aulas. Revista Mackenzie de Educação Física e Esporte, São Paulo, v. 5, n. 2, p. 13-19, 2006.

MINATTO, G.; RIBEIRO, R. R.; JUNIOR, A. A.; SANTOS, K. D. Idade, maturação sexual, variáveis antropométricas e composição corporal: influências na flexibilidade. Ver. Bras. cineantropom desempenho hum, 12(3), 151-8, 2010.

NASCIMENTO, C. P. A atividade pedagógica da educação física: a proposição dos objetos de ensino e o desenvolvimento das atividades da cultura corporal. 2014. Tese de Doutorado. Universidade de São Paulo.

RASSILAN, E. A.; GUERRA, T. C. Evolução da flexibilidade em crianças de 7 a 14 anos de idade de uma escola particular do município de Timóteo-MG. Movimentum, 1, 1-13, 2006.

REIS, T; SUSUKI, F. G. A utilização do Jiu-Jitsu brasileiro como conteúdo da Educação Física escolar. Revista Digital, Buenos Aires - ANO 17 - No 160 - junho de 2012.

RUFINO, L. G. B.; DARIDO, S. C. O jiu-jitsu brasileiro nas três dimensões dos conteúdos nas aulas de educação física escolar. In: IV colóquio de pesquisa qualitativa em motricidade humana: as lutas no contexto da motricidade, 4.; III simpósio sobre o ensino de graduação em educação física: 15 anos do curso de educação física da UFSCar; Shoto workshop, 5., 2009, São Carlos. Anais... São Carlos: UFSCar, 2009.

ULBRICH, A. Z.; BOZZA, R.; MACHADO, H. S.; MICHELIN, A.; VASCONCELOS, I. Q A.; NETO, A. S.; DE CAMPOS, W. Aptidão física em crianças e adolescentes de diferentes estágios maturacionais. Fitness \& performance journal, (5), 277-282, 2007.

WILLRICH, A; AZEVEDO, C. C. F de; FERNANDES, J. O. Desenvolvimento motor na infância: influência dos fatores de risco e programas de intervenção. Rev Neurocienc, v. 17, n. 1, p. 51-6, 2009.

\section{Como referenciar este artigo}

ALBERTO DA SILVA, Carlos; PAULA RODRIGUES, Abraham Lincoln de; LOPES SOARES, Stela. Comparação dos níveis de aptidão física entre escolares praticantes e não praticantes de jiu-jitsu. Temas em Educação e Saúde, Araraquara, v. 15, n. 1, p. 71-92, jan./jun., 2019. e-ISSN 2526-3471. DOI: 10.26673/tes.v15i1.12357

Submetido em: $12 / 12 / 2018$

Aprovado em: 30/03/2019 